\title{
Effet de la photopériode sur la production, la composition du lait et sur les consommations volontaires chez la brebis laitière
}

\author{
F Bocquier $^{1 *}$, S Ligios ${ }^{2}, \mathrm{G}_{\text {Molle}}^{2}$, $\mathrm{S} \mathrm{Casu}^{2}$ \\ 'Laboratoire de recherches sur la sous-nutrition des ruminants, Inra, \\ 63122 Saint-Genès-Champanelle, France ; \\ ${ }^{2}$ Istituto Zootecnico e Caseario per la Sardegna, 07040 Olmedo, Italie
}

(Reçu le 28 avril 1997 ; accepté le 20 juin 1997)

\begin{abstract}
Summary - Effect of photoperiod on milk yield, milk composition and voluntary food intake in lactating dairy ewes. Thirty-eight dairy ewes of the Sarda breed were divided in two groups which were equilibrated for zootechnical criteria. They were subjected to artificially controlled photoperiods of either long daylight (JL: $15 \mathrm{~h} 30 \mathrm{~L}, 8 \mathrm{~h} 30 \mathrm{D}$ ) or short daylight (JC: $8 \mathrm{~h} 30 \mathrm{~L}, 15 \mathrm{~h} 30 \mathrm{D}$ ). The experimental periods began 25 days before lambing and were maintained until day 150 of lactation. At this time the photoperiod was abruptly changed to equilibrated daylenght treatments (12h L, 12h D). Throughout the whole experiment, the ewes were fed alfalfa hay ad libitum as well as a constant proportion of a commercial concentrate (table I). JL ewes produced more milk $(+25.3 \%)$ than JC ewes (fig 3). After 150 days, the daylight increase $(+3 \mathrm{~h} 30 / \mathrm{exJC})$ made it possible to maintain milk yield at the initial value (only a $-8.0 \%$ drop between day 145 and day 187 ), while the daylight decrease $(-3 \mathrm{~h} 30 / \mathrm{exJL})$ resulted in a sharp drop in milk yield $(-37.7 \%)$ over the same period. The milk solid concentration from the JL ewes (fig 4) was less than that of the JC ewes. The maximum differences, which occurred between days 30 to 40 , were $14.2 \mathrm{~g} / \mathrm{litre}$ for fat content and $11.0 \mathrm{~g} / \mathrm{litre}$ for protein content. The rapid increase of daylength (exJC) was less favourable to milk solid concentration than its decrease (exJL) for both the fat content $(-7.8 \mathrm{~g} /$ litre $)$ and protein content $(-6.5 \mathrm{~g} / \mathrm{litre})$. The levels of voluntary food intake (fig 1), which were identical at the end of pregnancy, started being different on day 25 of lactation. Between days 28 and 150 the mean total DM consumption for the JL ewes was $16.3 \%$ higher than those of the JC ewes. After the daylength change, the intake differences were reduced and became stabilised at a mean value of $+6.2 \%$ for the exJL ewes. By the end of experiment, when the photoperiod had been the same for 30 days, the total food intake levels were very similar. Time scale effects of daylight in lactating ewes act primarily on milk yield, then on milk composition and finally on food intake. Refractoriness to photoperiod seems to appear after being subjected to constant light treatments for more than 100 days.
\end{abstract}

dairy ewe / photoperiod / milk composition / voluntary intake

* Correspondance et tirés à part

Tél : (33) 0473624062 ; fax : (33) 0473624519 ; e-mail : bocquier@clermont.inra.fr 
Résumé - Trente-huit brebis laitières de race Sarde ont été réparties dans deux lots équilibrés sur des critères zootechniques. Elles ont été soumises à une photopériode artificielle contrôlée : jours longs (JL : $15 \mathrm{~h} 30 \mathrm{~L}, 8 \mathrm{~h} 30 \mathrm{D}$ ) ou jours courts (JC : $8 \mathrm{~h} 30 \mathrm{~L}, 15 \mathrm{~h} 30 \mathrm{D}$ ) depuis $25 \mathrm{j}$ avant la mise bas jusqu'au $150^{\mathrm{e}}$ jour de la lactation. Les brebis des deux lots ont ensuite été soumises brutalement au même traitement photopériodique (12h L, 12h D). Pendant toute l'expérience, les brebis ont reçu ad libitum une ration à base de foin de luzerne et d'un aliment concentré du commerce dont la proportion a été maintenue constante dans la ration. Par rapport aux brebis en JC, celles qui étaient en JL ont eu une production laitière accrue en moyenne de $25,3 \%$. Après $150 \mathrm{j}$ de lactation, l'allongement de la durée d'éclairement pour les brebis en $\mathrm{JC}(+3 \mathrm{~h} 30)$ a permis de maintenir la production laitière brute à son niveau initial $(-8,0 \%)$, alors que pour les brebis en $\mathrm{JL}$, la diminution de la durée d'éclairement $(-3 \mathrm{~h} 30)$ a entraîné une forte baisse de la production laitière $(-37,7$ \% entre J 145 et J I 87). En JL les taux butyreux (TB) et protéique (TP) du lait ont eu tendance à baisser par rapport à ceux des brebis en JC : les écarts maximaux, qui apparaissent après 30 à $40 \mathrm{j}$, ont été de 14,2 g/litre pour le TB et de $11,0 \mathrm{~g} /$ litre pour le TP. L'augmentation brutale de la durée d'éclairement (exJC) a été défavorable par rapport à sa diminution (exJL), aussi bien pour le TB $(-7,8 \mathrm{~g} /$ litre) que pour le TP $(-6,5 \mathrm{~g} /$ litre $)$. Les quantités volontairement ingérées, qui étaient identiques en fin de gestation, se sont différenciées à partir du $25^{\mathrm{e}}$ jour de lactation. Ainsi, entre $\mathrm{J} 28$ et J150 les consommations totales moyennes des brebis en JL ont été de 16,3\% supérieures à celles des brebis en JC. Après le changement de photopériode, la différence d'ingestion a fortement diminué pour s'établir en moyenne à $+6,2 \%$ dans le lot JL par rapport au lot JC. En fin d'essai, lorsque les photopériodes étaient identiques depuis plus de $30 \mathrm{j}$, les consommations totales de MS ont été très proches pour les deux lots de brebis. Au total, la durée d'éclairement agit à différentes échelles de temps : d'abord sur le volume de lait, ensuite sur sa composition et enfin sur les quantités volontairement ingérées. Après $100 \mathrm{j}$ de traitement photopériodique constant il semble s'installer un état réfractaire aux effets de la photopériode.

\section{brebis laitière / photopériode / production et composition du lait / ingestion}

\section{INTRODUCTION}

En Sardaigne le lait de brebis étant destiné exclusivement à la fabrication fromagère (Pecorino Romano, Fiore Sardo...), il est important de connaître les facteurs de variation de sa composition chimique. Outre les facteurs bien connus comme le stade de lactation, l'alimentation (Bocquier et Caja, 1993) et l'état sanitaire, il semble que des facteurs liés à la saison aient également une influence sur le volume et la composition du lait produit (Casu, 1965 ; Casu et Marcialis, 1967). En effet, l'analyse des nombreuses courbes de lactations enregistrées en Sardaigne montre que les brebis qui mettent bas au printemps (durée du jour croissante) ont une production laitière plus importante que celles qui agnèlent à l'automne (Carta et al, 1995). De plus, lorsque l'effet du stade de lactation est pris en compte, il persiste un effet de la saison de mise bas (Carta et al, 1995). Dans tous les cas, il n'est pas possible d'isoler les différents facteurs du milieu (photopériode et température ambiante) qui varient simultanément et qui, en outre, influencent les caractéristiques de l'alimentation et en particulier le pâturage (Sitzia et al, 1997). Le but de cette expérimentation était de mettre en évidence, chez la brebis Sarde, l'influence de la photopériode sur la production et la composition du lait et sur les consommations volontaires lorsque l'alimentation est de composition constante. En effet, il a été montré (Bocquier, 1985) que la photopériode pouvait modifier fortement le volume et la composition du lait produit par des brebis laitières, alors que cet effet est très faible chez la vache laitière (Peters et al, 1978, 1981). 


\section{MATÉRIEL ET MÉTHODES}

\section{Choix des brebis}

Trente-huit brebis laitières de race Sarde du troupeau de l'IZCS (Istituto Zootecnico e Caseario per la Sardegna) ont été réparties dans deux blocs expérimentaux sur les critères zootechniques suivants : stade de gestation et nombre de fotus (échographie), production laitière antérieure, âge, poids vif et état corporel.

\section{Maîtrise de la photopériode}

Les brebis étaient logées dans deux pièces séparées et isolées de la lumière extérieure ; le renouvellement de l'air était réalisé par une ventilation active. L'éclairement était assuré dans chaque pièce par 16 tubes fluorescents au néon placés à $2,20 \mathrm{~m}$ du sol. L'intensité lumineuse mesurée au niveau des yeux des brebis était de 500 lux. La durée d'éclairement était programmée automatiquement.

L'essai a débuté le 19 octobre, lorsque la durée naturelle du jour ( $\mathrm{JN})$ était de $11 \mathrm{~h}(\mathrm{JN}$ : $11 \mathrm{~L}, 13 \mathrm{D}$ ). Progressivement, en $10 \mathrm{j}$, la durée quotidienne d'éclairement a été modifiée pour s'établir à $15 \mathrm{~h} 30$ pour les brebis placées en photopériode longue (JL : 15 h $30 \mathrm{~L}, 8 \mathrm{~h} 30 \mathrm{D})$ et à $8 \mathrm{~h} 30$ pour celles en photopériode courte (JC : $8 \mathrm{~h} 30 \mathrm{~L}, 15$ h 30 D). Les régimes photopériodiques, établis $25 \mathrm{j}$ avant la date moyenne de mise bas, ont été maintenus constants pendant les 150 premiers jours de lactation. Après $150 \mathrm{j}$ de lactation, les durées d'éclairement ont été brutalement amenées à l'équilibre : $12 \mathrm{~h}$ de lumière et $12 \mathrm{~h}$ d'obscurité par jour (JE : $12 \mathrm{~L}, 12 \mathrm{D}$ ). Les températures et hygrométries ambiantes ont été enregistrées automatiquement. Nous n'avons observé, en moyenne sur la durée de l'essai, aucune différence de température et d'hygrométrie entre les deux pièces expérimentales.

\section{Alimentation}

Les brebis étaient logées en lot sur caillebotis afin qu'il n'y ait pas de consommation de litière. Le foin a été distribué en deux repas par jour : après la traite du matin et avant celle du soir. L'aliment concentré a été distribué en salle de traite à $8 \mathrm{~h}$ et à $16 \mathrm{~h}$. Les quantités d'aliments offertes et refusées ont été pesées tous les jours. Pendant toute l'expérience, les brebis ont reçu une ration à base de foin de luzerne ad libitum (distribution ajustée à $15 \%$ de refus) et d'un aliment concentré du commerce comportant également des minéraux et des vitamines. Pour maintenir un rapport concentré-fourrage identique dans les deux lots, les apports d'aliment concentré ont été ajustés sur les consommations de foin. Ce rapport concentré-fourrage a été maintenu à $30 \%$ au cours des 100 premiers jours de lactation, il a ensuite été amené à $15 \%$ pendant le reste de l'expérience. La digestibilité des rations a été mesurée sur cinq brebis Sardes taries et non gravides placées en photopériode naturelle. Ceci a permis d'estimer les valeurs nutritionnelles des rations (tableau I).

Tableau I. Caractéristiques chimiques et valeur alimentaire déterminées par les digestibilités in vivo des rations sur brebis taries avant ou après $100 \mathrm{j}$ de lactation.

\begin{tabular}{|c|c|c|c|c|c|c|c|c|c|c|}
\hline \multirow[b]{2}{*}{ Rations } & \multicolumn{4}{|c|}{ Analyse chimique } & \multicolumn{3}{|c|}{ Digestibilité } & \multicolumn{3}{|c|}{ Valeur nutritive $(/ \mathrm{kg} M S$} \\
\hline & $\begin{array}{c}M S \\
(\% M S)\end{array}$ & $\begin{array}{c}M O \\
(\% M S)\end{array}$ & $\begin{array}{c}\text { MAT } \\
\text { (\%MS) }\end{array}$ & $\begin{array}{c}C B \\
(\% M S)\end{array}$ & $\begin{array}{c}M O \\
(\% M S)\end{array}$ & $\begin{array}{c}\text { MAT } \\
(\% M S)\end{array}$ & $\begin{array}{c}C B \\
(\% M S)\end{array}$ & $U F L$ & $\begin{array}{c}\text { PDIN } \\
(g)\end{array}$ & $\begin{array}{c}P D I E \\
(g)\end{array}$ \\
\hline Foin de luzerne $($ lac $<100 \mathrm{j})$ & 85 & 88 & 28 & 29 & - & - & - & 0,70 & 1.33 & 102 \\
\hline Foin de luzerne (lac $>100 \mathrm{j})$ & 86 & 90 & 18 & 34 & - & - & - & 0,65 & 117 & 89 \\
\hline Aliment concentré $(\mathrm{C})$ & 89 & 91 & 18 & 10 & - & - & _- & 0,98 & 117 & 104 \\
\hline Ration $70 \%$ lac $<100$ jet $30 \% \mathrm{C}$ & C 85 & 89 & 20 & 23 & 67 & 70 & 41 & 0,79 & 120 & 103 \\
\hline Ration $85 \%$ lac $>100$ jet $15 \% \mathrm{C}$ & 84 & 89 & 18 & 30 & 60 & 66 & 45 & 0,74 & 131 & 102 \\
\hline
\end{tabular}

Pour le foin de luzerne, les consommations étaient de $1,06 \mathrm{kgMS}$ de foin seul, de $1,4 \mathrm{kgMS}$ pour la ration à $15 \% \mathrm{C}$ et de $1,8 \mathrm{kgMS}$ pour la ration à $30 \% \mathrm{C}$.

Lac, lactation. 


\section{Conduite des brebis}

Les brebis ont été traites mécaniquement deux fois par jour après le sevrage des agneaux qui a eu lieu 2 à $3 \mathrm{j}$ après leur naissance. Elles ont été tondues $166 \mathrm{j}$ après la mise bas. Les productions laitières individuelles brutes (PLB), ainsi que la composition chimique du lait (taux butyreux [TB] et protéique [TP]), ont été mesurées une fois par semaine. Pour tenir compte des différences de composition chimique du lait, nous avons exprimé la production en lait standardisé (PLS) sur la base d'une valeur énergétique constante (5 MJ/litre ; Bocquier et al, 1993). Des pesées et des estimations de l'état corporel (méthode de Russel et al, 1969) des brebis ont été effectuées juste après la mise bas puis toutes les deux semaines à heure fixe.

\section{Traitement statistique des données}

Les résultats ont été analysés avec le programme Sas (1988) en utilisant la procédure univariate split plot appliquée à des mesures répétées. Les deux périodes expérimentales situées de part et d'autre du changement de photopériode : P I ( $149 \mathrm{j})$ et $\mathrm{P} 2(41 \mathrm{j})$ ont été considérées séparément. Dans le modèle, l'effet de la photopériode (JL vs JC) a été associé à l'effet brebis à l'intérieur de chaque niveau photopériodique, au numéro du contrôle laitier ( $\left.\mathrm{N}^{\circ} \mathrm{CTRL}\right)$ et à l'interaction numéro de contrôle photopériode. L'effet de la photopériode a été testé sur le terme d'erreur de l'effet brebis à l'intérieur du traitement photopériodique : $\mathrm{Y}=\mathrm{PHOTO}+\mathrm{BREBIS}$ (PHOTO) $+\mathrm{N}^{\circ} \mathrm{CTRL}+\mathrm{N}^{\circ} \mathrm{CTRL} * \mathrm{PHOTO}+$ erreur.

\section{RÉSULTATS \\ Évolution des consommations d'aliments}

Avant la mise bas, les consommations moyennes volontaires de foin ont été identiques pour les deux traitements photopériodiques (fig I). Juste après l'agnelage et au cours des 4 premières semaines de lactation, les consommations totales ont encore été semblables dans les deux lots (fig 1).

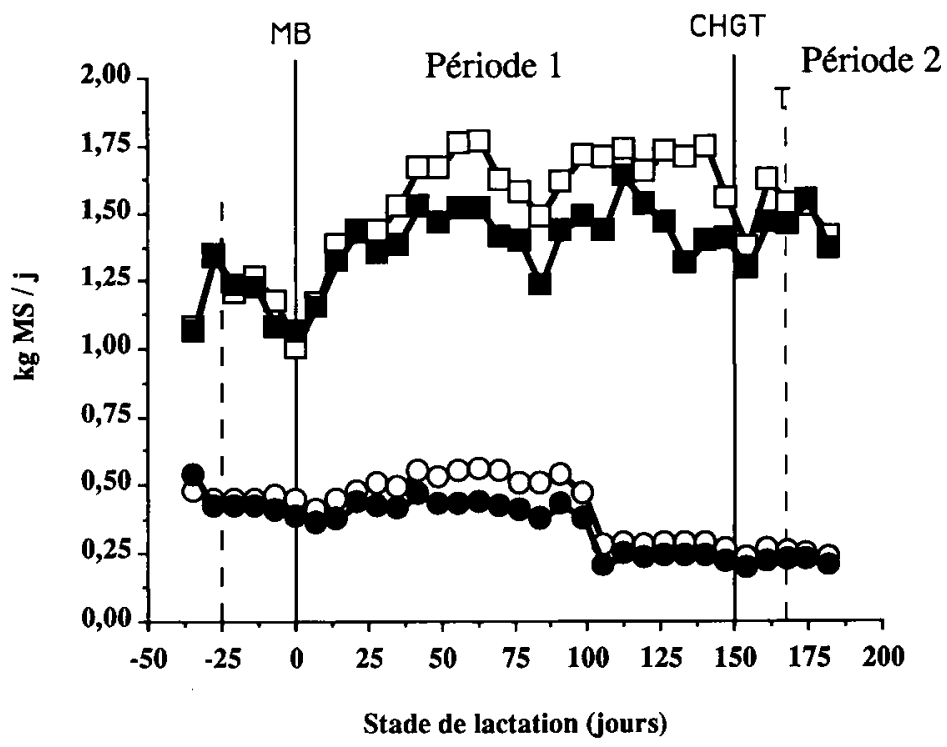

Fig 1. Évolutions des consommations de foin $(\square, \square)$ et d'aliment concentré $(\bigcirc, \bullet)$ en fonction de la photopériode (longue $\square, \bigcirc$; courte $\square, 0$ ). MB : mise bas ; CHGT : changement de photopériode ; $\mathrm{T}$ : tonte des brebis. 
Après ces 4 semaines et jusqu'au changement de photopériode ( $150 \mathrm{j}$ ), les brebis placées en jours longs ont accru progressivement leur consommation d'aliments par rapport à celles qui étaient en photopériode courte : entre J28 et J150 les consommations totales moyennes des brebis en JL ont été de $2,1 \mathrm{~kg} \mathrm{MS} /$ jour alors qu'en JC elles étaient $1,8 \mathrm{~kg} \mathrm{MS} /$ jour (soit un écart de $+16 \%$ ). L'écart maximum de consommation $(+28 \%)$ se situe $140 \mathrm{j}$ après la mise bas, soit $10 \mathrm{j}$ avant le changement de la durée d'éclairement. Après le changement de photopériode, la différence d'ingestion a fortement diminué pour s'établir en moyenne à $+6 \%$ pour les brebis précédemment placées en photopériode longue $(1,75 \mathrm{~kg}$ MS/jour) par rapport à celles placées en photopériode courte $(1,65 \mathrm{~kg}$ $\mathrm{MS} / \mathrm{jour}$ ). En fin d'essai, lorsque les photopériodes étaient identiques depuis plus de $30 \mathrm{j}$, les consommations de MS totales ont été très proches pour les brebis des deux lots et à $174 \mathrm{j}$ elles sont de $1,78 \mathrm{~kg}$ MS/jour en exJL (diminution de la durée du jour) comme en exJC (augmentation de la durée du jour). Les fluctuations d'ingestion, qui apparaissent simultanément dans les deux traitements, sont vraisemblablement dues aux variations de qualité du fourrage.

\section{Évolution du poids et de l'état corporel des brebis}

Le poids vif et l'état corporel moyen des brebis des deux lots étaient très proches avant que ne débute le traitement photopériodique (fig 2 ). Après la mise bas ( $12 \mathrm{j}$ ) les poids vifs moyens des brebis des deux lots étaient encore très proches ( $\mathrm{JC}=49,8 \mathrm{vs}$ $\mathrm{JL}=50,2 \mathrm{~kg} ; \mathrm{ns})$. Ensuite, les poids vifs des brebis placées en jours courts restent pratiquement constants (fig 2), alors qu'en jours longs ils augmentent régulièrement : au moment du changement de photopériode (150 j) l'écart de poids vif, bien que non significatif, était de $+3,3 \mathrm{~kg}$ (soit $6,9 \%$ ).
Cet écart se maintient ensuite jusqu'à la fin de l'expérience et ne semble pas affecté par la modification de la durée d'éclairement.

L'état corporel moyen des brebis est identique (note de 2,99 points) $7 \mathrm{j}$ avant mise bas dans les deux lots ; il reste très stable et proche entre les traitements photopériodiques (ns) au cours de l'expérimentation (fig 2). En début de lactation de légères différences (ns) apparaissent en faveur des brebis en JC par rapport à celles en JL (respectivement 2,99 vs 2,88 à J28).

La photopériode longue, appliquée moins d'un mois avant la mise bas, n'a pas eu d'influence (ns) sur le poids des agneaux à la naissance, qu'il s'agisse des agneaux nés simples (JL : 3,13 vs JC : 2,91 kg) ou doubles (JL : $2,57 \mathrm{vs} \mathrm{JC}: 2,53 \mathrm{~kg}$ ). De plus, la prolificité des brebis des deux lots était comparable (JL : 1,45 et JC : 1,57 ).

\section{Production et composition du lait}

Dès le premier contrôle laitier, les brebis en photopériode longue produisent environ $20 \%$ plus de lait que celles qui sont en JC. Après $50 \mathrm{j}$ de lactation cette différence s'estompe en valeur absolue (fig 3), mais reste proportionnelle à la production laitière. Ainsi, durant les 150 premiers jours de lactation $(\mathrm{Pl})$, les brebis placées en photopériode longue ont produit en moyenne significativement $(p<0,03)$ plus de lait $(1,21$ litre/jour) que celles qui étaient en jours courts $(0,96$ litre/jour $)$, soit un écart relatif de $+25 \%$ (fig 3 ). Le changement brutal de durée d'éclairement a eu des effets opposés selon que la durée du jour s'est accrue (exJC) ou a diminué (exJL). En effet, pour les brebis qui étaient en JC depuis $175 \mathrm{j}$, l'accroissement de la durée d'éclairement a conduit à une stabilisation de la production laitière qui s'est maintenue pendant $37 \mathrm{j}$ à 0,596 litre/jour (fig 3). À l'inverse, chez les brebis dont la durée d'éclairement a été diminuée de $3 \mathrm{~h} 30$, la production laitière a 


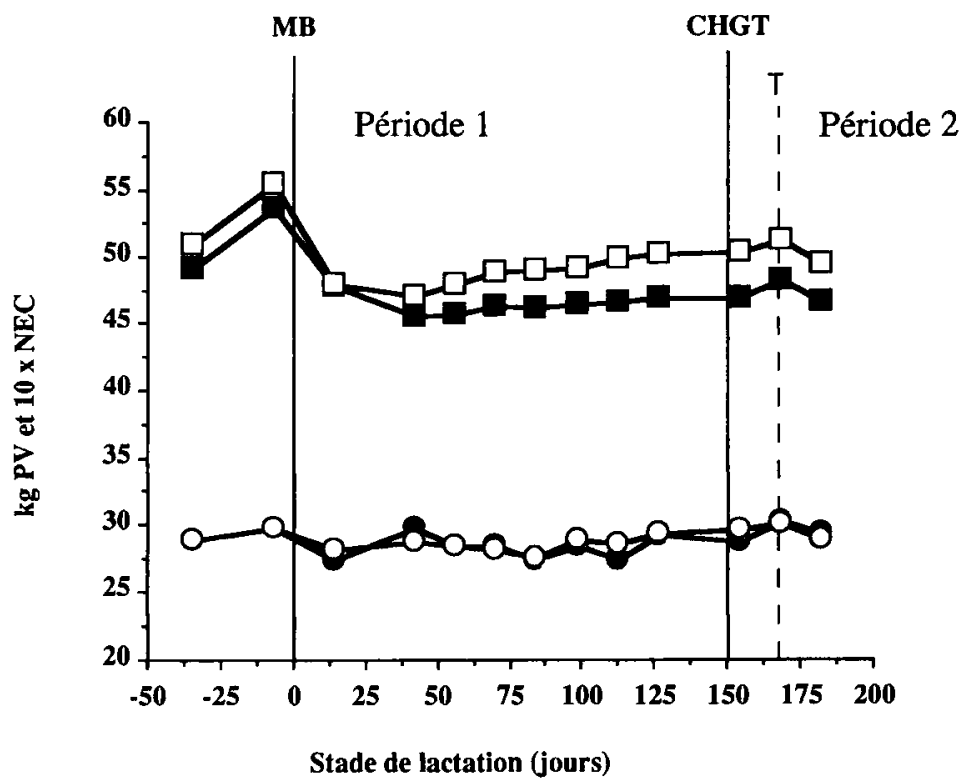

Fig 2. Évolution du poids vif $(\boldsymbol{\square}, \square)$ et de la note d'état corporel des brebis $(\bigcirc, \bullet)$ selon la photopériode (longue $\square, \bigcirc$ ou courte $\mathbf{\square}, \bigcirc$ ). MB : mise bas ; CHGT : changement de photopériode ; $\mathrm{T}$ : tonte des brebis.

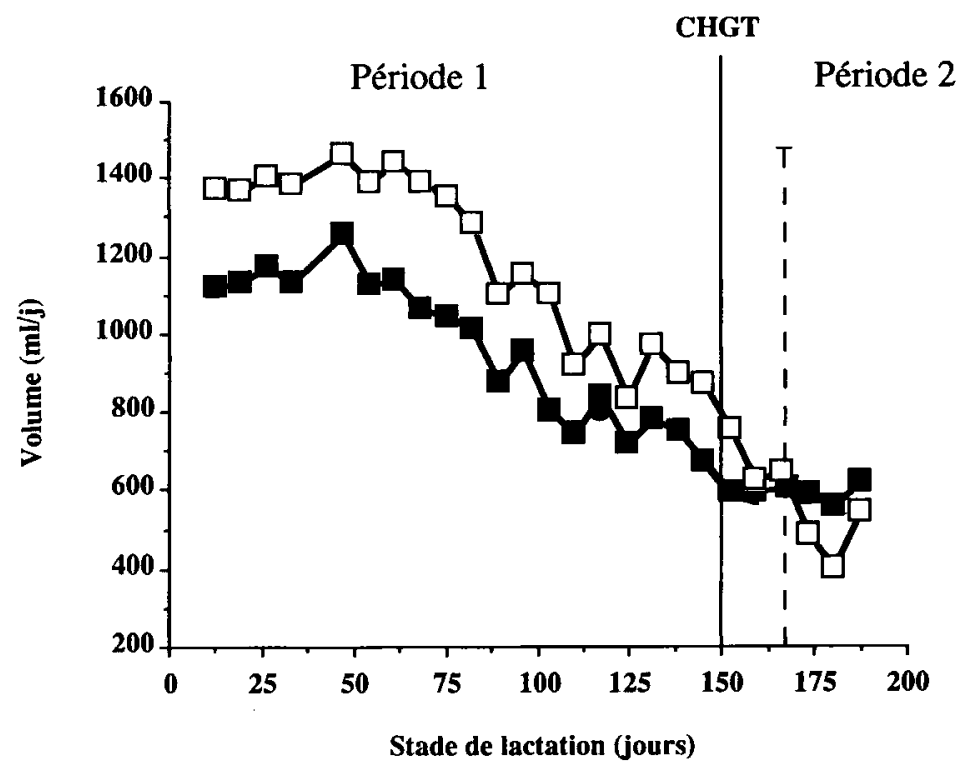

Fig 3. Évolution de la production laitière brute de brebis placées en photopériode longue $(\square)$ ou courte (ם). CHGT : changement de photopériode ; T : tonte des brebis. 
chuté fortement : au cours des $30 \mathrm{j}$ qui ont suivi le changement de photopériode, la production laitière brute a diminué de 0,468 litre et a atteint un niveau minimal de 0,406 litre/jour (fig 3). Au total, sur les 37 j qui suivent le changement de photopériode, les productions laitières moyennes ont été pratiquement égales dans les deux lots : exJL $=0,579$ litre/jour et exJC $=0,596$ litre/jour.

Les taux butyreux et protéique du lait étaient très proches en début de lactation (au $10^{\mathrm{e}}$ jour, $\mathrm{TB}: \mathrm{JL}=61,9$ vs $\mathrm{JC}=62,2$ $\mathrm{g} /$ litre et $\mathrm{TP}: \mathrm{JL}=51,6 \mathrm{vs} \mathrm{JC}=54,6 \mathrm{~g} /$ litre) et les écarts de composition du lait (fig 4) ont été observés surtout au cours des 100 premiers jours de lactation avec un maximum au $30^{\mathrm{e}}$ jour : TB $=-14,2 \mathrm{~g} /$ litre (ns) et $\mathrm{TP}=-11,0 \mathrm{~g} / \mathrm{litre}(\mathrm{ns})$. Après $100 \mathrm{j}$, ces écarts se sont estompés (fig 4). Globalement, le taux butyreux moyen du lait est légèrement (ns) plus faible en photopériode longue $(60,8 \mathrm{~g} /$ litre) qu'en photopériode courte $(62,7 \mathrm{~g} / \mathrm{litre})$. De même, c'est en photopériode longue que le taux protéique moyen du lait est le plus faible $(52,0 \mathrm{vs}$ $54,3 \mathrm{~g} /$ litre $; p<0,09$ ).

Juste avant le changement de durée d'éclairement, les taux butyreux $(\mathrm{TB}=$ $66,1 \mathrm{~g} /$ litre) et protéique ( $\mathrm{TP}=58,8 \mathrm{~g} /$ litre) étaient identiques (fig 4 ) dans les deux traitements. Ces taux se sont accrus dans les deux lots et ont été maximum au $23^{\mathrm{e}}$ jour après le changement ( $\mathrm{TB}=+17,5 \mathrm{~g} /$ litre et $\mathrm{TP}=+8,5 \mathrm{~g} / \mathrm{litre}$ ), puis ont diminué pour atteindre respectivement les valeurs de 74,0 et $63,1 \mathrm{~g} /$ litre en fin d'expérience. De plus, les brebis pour lesquelles la photopériode a été accrue (exJC) ont produit en moyenne, au cours des $30 \mathrm{j}$ qui ont suivi le changement, un lait moins riche que celles dont la durée d'éclairement a été diminuée (exJL) : qu'il s'agisse du TB (exJC $=69,7$ g/litre vs exJL $=77,5 \mathrm{~g} /$ litre $: p<0,001)$ ou du

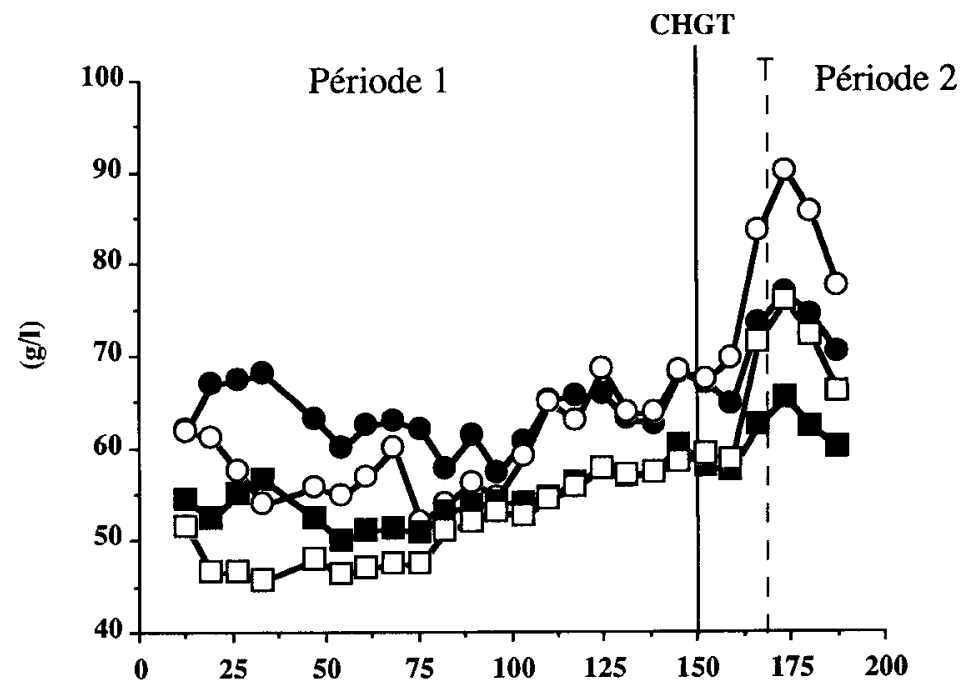

Stade de lactation (jours)

Fig 4. Évolutions de la composition chimique du lait de brebis en fonction de la photopériode (longue $\square, \bigcirc$; courte $\square, 0)$ : variations du taux butyreux $(\bigcirc, \bullet)$ et du taux protéique $(\boldsymbol{\square}, \square)$. CHGT : changement de photopériode ; $\mathrm{T}$ : tonte des brebis. 
$\mathrm{TP}(\mathrm{exJC}=60,5 \mathrm{~g} /$ litre vs exJL $=66,3$ g/litre : $p<0,001)$. L'écart maximal de composition chimique du lait entre les deux traitements photopériodiques apparaît au $23^{\mathrm{e}}$ jour après le changement : $\mathrm{TB}=-13,1 \mathrm{~g} /$ litre et TP $=-10,5 \mathrm{~g} /$ litre.

Malgré la dilution du lait en matières utiles chez les brebis en JL, la production laitière moyenne au cours de la première période, exprimée en lait standard, est plus élevée chez les brebis en JL (JL $=1,074$ litre/jour vs JC $=0,891$ litre/jour), soit une différence de $20,6 \%$ entre les deux traitements photopériodiques $(p<0,05)$. Après modification de la durée d'éclairement, malgré les fortes variations de la composition du lait, les productions laitières standardisées sont restées (ns) plus élevées pour les brebis précédemment en JL (0,602 g/litre) par rapport à celles en JC (0,586 litre/jour).

\section{DISCUSSION}

Cette expérience avait pour objectif principal de vérifier que la photopériode exerce une influence sur les performances laitières de brebis de race Sarde et qu'une partie de ces effets peuvent s'expliquer par des différences de quantités ingérées. Mauléon et Rougeot (1962) ont montré que la période de reproduction des brebis est dépendante de la photopériode, mais qu'il existe de fortes différences de sensibilité entre races. Il semble que, globalement, les races natives des régions les plus proches des pôles (Soay, Texel) soient plus sensibles à la durée d'éclairement que celles issues des zones plus équatoriales (Yeates, 1949). Ceci est cohérent avec l'amplitude de la variation de durée du jour en fonction de la latitude. Pour Ortavant et al (1988) la photopériode serait le signal principal (proximal) qui conditionne la mise en place de la reproduction à une période qui, compte tenu de la durée de gestation, s'avère être la plus favorable, aux plans alimentaire et climatique, à la survie et la croissance des jeunes. La photopériode exercerait également un rôle secondaire (distal) en modifiant l'ingestion d'aliments, la production laitière, l'utilisation des réserves corporelles et la croissance des jeunes, ce qui concourt à l'adaptation des ovins à leur milieu. Cependant, ce rôle distal de la photopériode (Ortavant et al,1988) n'est basé que sur un faisceau convergent de preuves expérimentales qui ont été obtenues sur différents types d'ovins (agneaux, brebis, mâles castrés ou entiers), avec différents modes d'alimentation (limité ou à volonté, fourrages seuls ou complémentés). L'expérience que nous avons réalisée permet de montrer que la photopériode a une influence sur la lactation des brebis, avec des modifications des consommations alimentaires et de composition du lait.

\section{Photopériode et production laitière brute}

L'allongement artificiel de la photopériode exerce une influence réduite sur la production laitière des bovins $(+10 \%$ : Peters et al, $1978 ;+6 \%$ : Peters et al, 1981) et des caprins ( $<3 \%$; Terqui et al, 1983), mais une influence assez forte chez les ovins (race Préalpes) à la traite $(+52 \%$ et $+30 \%$; Bocquier et al, 1986). Pour cela il faut que les régimes lumineux soient établis longtemps avant la mise bas ( $-42 \mathrm{j}$ : Bocquier, 1985), car s'il ne sont établis qu'au moment de la mise bas ils n'affectent pas le démarrage de la lactation (Bocquier et al, 1990). Pour les brebis Sardes de l'essai, avec des régimes lumineux établis $25 \mathrm{j}$ avant mise bas, la production laitière est accrue par la photopériode longue dans une proportion plus faible $(+25,3 \%)$ que celle rapportée lorsque les traitements sont établis $42 \mathrm{j}$ avant mise bas $(+52 \%$ et $+30 \%$; Bocquier et al, 1986).

L'inversion brutale des traitements lumineux (JC vs JL) en cours de lactation modifie l'évolution de la production laitière, fortement chez la brebis $(+34$ et $+12 \%$; Bocquier, 1985) et dans une moindre mesure 
chez la vache (Peters et al, 1978). Cependant, les essais réalisés ne permettaient pas d'isoler l'effet du changement de photopériode de celui de la durée d'éclairement. En revanche, pour le présent essai, la photopériode étant identique (12L: 12D) après changement de la durée d'éclairement $( \pm 3 \mathrm{~h} 30)$, il a été possible d'isoler l'effet propre de ce changement. Nous montrons que le changement de la durée d'éclairement a un effet important $(-8 \%$ (exJC) ou $-37,7 \%$ (exJL)) sur la persistance de la lactation qui varie alors dans le même sens que la variation de la durée du jour.

\section{Photopériode et composition du lait}

En jours longs, les taux butyreux et protéique du lait ont tendance à diminuer chez les brebis Sardes comme chez les brebis Préalpes (Bocquier, 1985 ). Dans ces deux races, l'écart maximal de TB apparaît entre le $30^{\mathrm{e}}$ et le $40^{\mathrm{e}}$ jour de lactation (Sarde : $-14,2$ g/litre vs Préalpes : - 10,0 g/litre). En ce qui concerne le TP, l'effet est plus marqué chez les Sardes $(-11 \mathrm{~g} /$ litre $)$ que chez les Préalpes ( $-3 \mathrm{~g} / \mathrm{litre})$. Bien que les brebis de ces deux races aient été soumises à des traitements photopériodiques de même durée, les différences de taux observées sont confondues avec les niveaux laitiers et avec les conduites alimentaires : les Sardes étant nourries ad libitum alors que les Préalpes étaient en alimentation limitée.

Après changement des durées d'éclairement, il est observé un accroissement transitoire et spectaculaire de la teneur en matières utiles du lait dans tous les cas. Cet effet n'a pas, à notre connaissance, été rapporté dans la bibliographie. Il n'a pas d'origine alimentaire car les consommations n'ont pas changé à court terme et aussi parce que, le plus souvent, les effets alimentaires affectent de façon opposée les deux taux (Bocquier et Caja, 1993). Dans cette situation, l'allongement de la durée d'éclairement provoque une baisse du TB
$(-7,8 \mathrm{~g} /$ litre $)$ et du TP $(-6,5 \mathrm{~g} /$ litre $)$ par rapport au lait des brebis soumises à une diminution de la durée d'éclairement. À l'issue d'une inversion complète des traitements lumineux en cours de lactation (Bocquier, 1985), il avait été observé des écarts de $-19,1 \mathrm{~g} /$ litre sur le TB et de $-13,5 \mathrm{~g} /$ litre pour le TP respectivement entre les lots soumis à des $\mathrm{JL}$ et ceux soumis à des JC. Le présent essai sur les brebis Sardes permet de mettre en évidence que la variation de la durée du jour peut, par elle-même, avoir des effets sur la composition du lait. Cet effet représente environ la moitié de celui observé à long terme lorsque les traitements lumineux sont inversés et maintenus constants.

Après le changement de photopériode $(\mathrm{J}+150)$, la variation brutale de la durée du jour provoque à $\mathrm{J}+165$ un accroissement des taux butyreux et protéiques du lait. Cette tendance, qui se prolonge jusqu' au $23^{\mathrm{e}}$ jour après inversion des régimes lumineux, est peut être un effet indirect de la tonte des brebis $(\mathrm{J}+\mathrm{l} 66)$. La tonte, en améliorant transitoirement le confort thermique des brebis, a sans doute permis d'accroître la concentration du lait. En effet, il a été montré (Bocquier, 1985) qu'une diminution de température ambiante de $10^{\circ} \mathrm{C}$ (de 28 à $18^{\circ} \mathrm{C}$ ) provoque un accroissement de la teneur en matières utiles du lait. L'effet favorable de la tonte a pu se manifester car en P2 la température ambiante était assez élevée pour des brebis $\left(23,5 \pm 2,7^{\circ} \mathrm{C}\right)$ et très supérieure à celle mesurée en $\mathrm{P} 1\left(13,5 \pm 2,6^{\circ} \mathrm{C}\right)$. Les résultats obtenus dans cet essai confirment (Bocquier, 1985) que les effets de la température ambiante et de la photopériode s'additionnent pour moduler la richesse du lait des brebis traites, même s'il s'agit ici d'un effet rémanent de la photopériode.

\section{Photopériode et ingestion d'aliments}

Les consommations volontaires d'aliments par les ovins sont accrues par l'allongement de la durée d'éclairement, qu'il s'agisse de 
jeunes $(+21 \%:$ Blaxter et al, 1982) ou d'adultes (Tarttelin, 1968) et de mâles castrés $(+45 \%$ : Gordon, $1964 ;+11 \%$ : Milne et al, $1976 ;+35 \%$ : Milne et al, $1978 ;+41 \%$ : Michalet-Doreau et Gatel, 1983). Cet effet de la photopériode est d'autant plus marqué que les rations sont plus ingestibles $(+11 \%$ avec du foin de fétuque et $+35 \%$ avec de l'herbe déshydratée : Milne et al, $1976 ; 1978)$. Les différences raciales de sensibilité à la photopériode sont importantes entre des béliers Soay $(+71 \%)$ et des béliers Suffolk (+ $52 \%$; Kay, 1979). Peu d'expérimentations ont été conçues pour mettre en évidence le temps nécessaire à la mise en place de l'effet de la photopériode sur l'ingestion. Néanmoins, lorsque la photopériode évolue cycliquement (cycle annuel, ou semestriel), le niveau d'ingestion maximal se produit entre 45 et $90 \mathrm{j}$ après le maximum de la durée d'éclairement (60 j/Soay et $90 \mathrm{j} / \mathrm{Suf}$ folk $\times$ Finn Dorset : Kay, 1979 ; 45 j/Texel : Michalet-Doreau et Gatel, 1983). Nous observons, chez la brebis laitière Sarde, que les quantités volontairement ingérées ne se différencient qu'à partir de $25 \mathrm{j}$ de lactation.
Or comme la photopériode a été modifiée $25 \mathrm{j}$ avant la mise bas, le délai d'apparition des effets de la photopériode $(50 \mathrm{j})$ est tout à fait conforme aux délais rapportés ci-dessus. Des écarts de consommations aurait pu apparaître plus tôt puisque les productions laitières sont différentes dès le début de lactation. Cependant il a été montré, chez des brebis allaitantes, que 1'accroissement des quantités ingérées en début de lactation est fortement dépendant de la nature des fourrages et assez peu dépendant du niveau de production laitière (Bocquier et al, 1987). Ce n'est qu'après le pic de lactation qu'il existe une relation étroite entre les quantités ingérées et la production laitière (Bocquier et Caja, 1993). Ceci peut expliquer qu'au cours des 6 premières semaines il n'y ait pas de différence d'ingestion entre traitements photopériodiques. Ultérieurement, il n'est plus possible de déterminer si les écarts de consommation en faveur des brebis soumises aux jours longs sont dus à la photopériode ou induits par les différences de production laitière. Lorsque la durée d'éclairement varie brutalement (150 j), les écarts de consommation s'amenuisent quand

Tableau II. Consommation alimentaire, apports énergétiques, protéiques et taux de couverture des besoins par brebis et par jour (moyennes par lots : hypothèses Inra, 1989)

\begin{tabular}{|c|c|c|c|c|c|c|c|c|}
\hline \multirow{2}{*}{$\begin{array}{l}\text { Période } \\
(\text { en j/MB) }\end{array}$} & \multirow{2}{*}{$\begin{array}{c}\text { Traitement } \\
\text { photo- } \\
\text { périodique }\end{array}$} & \multicolumn{3}{|c|}{ Consommation et apports } & \multirow{2}{*}{$\begin{array}{l}\text { Production } \\
\text { laitiere } \\
\text { standard } \\
\text { (litre/jour) }\end{array}$} & \multirow{2}{*}{$\begin{array}{c}\text { Poids vif } \\
\text { moyen } \\
(k g)\end{array}$} & \multicolumn{2}{|c|}{ Couverture des besoins } \\
\hline & & $k g M S$ & $U F L$ & $P D I^{u}$ & & & $U F L(\%)$ & $P D I(\%)$ \\
\hline \multicolumn{9}{|c|}{ De 1 à $28 \mathrm{j}$} \\
\hline & jours courts & 1,628 & 1,29 & 169 & 1,051 & 48,0 & 95 & 111 \\
\hline & jours longs & 1,673 & 1,32 & 174 & 1,237 & 48,1 & 89 & 108 \\
\hline \multicolumn{9}{|c|}{ De 29 à $149 \mathrm{j}$} \\
\hline & jours courts & 1,802 & 1,39 & 186 & 0,866 & 46,4 & 116 & 141 \\
\hline & jours longs & 2,096 & 1,62 & 216 & 1,013 & 48,9 & 122 & 145 \\
\hline \multicolumn{9}{|c|}{ De 150 à $190 \mathrm{j}$} \\
\hline & jours courts & 1,446 & 1,09 & 150 & 0,562 & 47,4 & 109 & 139 \\
\hline & jours longs & 1,760 & 1,30 & 180 & 0,538 & 50,4 & 129 & 158 \\
\hline
\end{tabular}

a PDI : PDIE limitants ; MB : mise bas. 
les productions laitières se rapprochent. Ils disparaissent après $30 \mathrm{j}$ sans que l'on puisse déterminer si la photopériode a exercé un effet dans ce laps de temps.

Chez la vache, qui est peu sensible à la photopériode, l'écart d'ingestion $(6 \%)$ entre animaux placés sur deux traitements lumineux couvre l'augmentation des besoins qui découlent de la variation de la production laitière $(+7 \%$; Peters et al, 1978). La même situation se produit chez la brebis Sarde avec une amplitude plus grande. À long terme, il ne semble pas que la régulation des quantités ingérées soit uniquement sous la dépendance de la satisfaction des besoins. En effet, malgré l'accroissement de leurs besoins, les brebis en JL ont des taux de satisfaction des besoins énergétiques plus élevés (tableau II) que celles qui sont en JC. Ces résultats sont originaux et complètent ceux qui avaient été obtenus sur des brebis Préalpes (Bocquier et al, 1986) pour lesquelles il avait été montré qu'à même quantités ingérées les brebis en JL orientaient plus d'énergie vers la production laitière au dépend de la reconstitution des réserves corporelles.

\section{CONCLUSIONS}

En ce qui concerne la production laitière brute, les résultats présents confirment, chez des brebis nourries ad libitum, l'effet favorable d'une photopériode longue et/ou d'un accroissement brutal de la durée d'éclairement. En début de lactation, la composition du lait n'est modifiée qu'après quelques jours, alors qu'un changement brutal de la durée d'éclairement modifie très rapidement et fortement la composition du lait en fin de lactation. Le présent essai a permis de montrer que la brebis perçoit non seulement la durée mais également la variation de la durée d'éclairement. À l'inverse, lorsque la durée d'éclairement est maintenue constante pendant longtemps (175 j), les effets de la photopériode sur la production et surtout sur la composition du lait ont tendance à s'estomper et il semble s'installer, comme pour la reproduction (Malpaux et al, 1996), un état réfractaire. Il est probable qu'en situation naturelle ces deux composantes de la photopériode (durée et/ou variation) influencent le volume et la composition du lait. Nous montrons également que chez les ovins en production l'ingestion volontaire est influencée par la durée d'éclairement mais à plus long terme. En situation naturelle il est difficile de quantifier comme nous l'avons fait l'influence des facteurs du milieu qui agissent pourtant de façon très importante sur la production laitière des brebis Sarde. Ces facteurs, ajoutés aux facteurs d'élevage, expliquent sans doute la très grande variabilité des performances laitières des brebis élevées au pâturage.

\section{REMERCIEMENTS}

Nous remercions G Nuvoli et G Scanu pour avoir effectué les analyses chimiques et A Carta pour son aide à l'analyse statistique des données.

\section{RÉFÉRENCES}

Blaxter KL, Fowler VR, Gill JC (1982) A study of the growth of sheep to maturity. J Agric Sci Camb 98 , $405-420$

Bocquier F (1985) Influence de la photopériode et de la température sur certains équilibres hormonaux et sur les performances zootechniques de la brebis en gestation et en lactation. Thèse doc ing sci agro, Ina-PG, $105 \mathrm{p}$

Bocquier F, Caja G ( 1993) Recent advances on nutrition and feeding of dairy sheep. Proc fifth Int Symp on Machine Milking of Small Ruminants, Budapest, May 14-20. Hungarian J Anim Prod 580-607

Bocquier F, Barillet F, Guillouet P, Jacquin M (1993) Prévision de l'énergie du lait à partir de différents résultats d'analyses : proposition de lait standard pour les brebis laitières. Ann Zootech 42, 57-66

Bocquier F, Kann G, Thériez M (1990) Relationships between secretory patterns of growth hormone, prolactin and body reserves and milk yield in dairy ewes under different photoperiod and feeding conditions. Anim Prod 51, 115-125

Bocquier F, Thériez M, Brelurut A (1987) The voluntary hay intake of ewes during the first weeks of lactation. Anim Prod 44, 387-394 
Bocquier F, Thériez M, Kann G, Delouis C (1986) Influence de la photopériode sur la partition de l'énergie nette entre la production laitière et les réserves corporelles chez la brebis laitière. Reprod Nutr Develop 26, 389-390

Carta A, Sanna SR, Casu S (1995) Estimating lactation curves and seasonal effects for milk, fat and protein in Sarda dairy sheep with a test day model. Livest Prod Sci 44, 37-44

Casu S, Marcialis A (1967) Ricerche sulla composizione del latte di pecora di razza Sarda : Variazioni della ripartizione dei costituenti azotati del latte. Bollettino Associazione Italiana Tecnici del Latte. Scienza e Tecnica Lattiero-Casearia, XVIII, 6, $427-436$

Casu S (1965) Ricerche sulla composizione del latte di pecora di razza Sarda. I. Alcuni fattori di variazione della percentuale di grasso nel latte. Bollettino della Societa Italiana di Biologia Sperimentale, XLI, 19, 1125-1128

Gordon JG (1964) Effect of the time of the year on the roughage intake of housed sheep. Nature 204 , $798-799$

Inra (1989) Ruminant Nutrition Recommended Allowances and Feed Tables (Jarrige R, ed) Inra-John Libbey Eurotext, Paris

Kay RNB (1979) Seasonal changes of appetite in deer and sheep. ARC Res Rev 5, 13-15

Malpaux B, Viguié C, Thièry JC, Chemineau P (1996) Contrôle photopériodique de la reproduction. Inra Prod Anim 9, 9-23

Mauléon P, Rougeot J (1962) Régulation des saisons sexuelles chez les brebis de races différentes au moyen de divers rythmes lumineux. Ann Biol Anim Bioch Biophys 2, 209-222

Michalet-Doreau B, Gatel F (1983) Évolution au cours d'une année des quantités de foin ingérées par des béliers castrés. Ann Zootech 32, 459-464
Milne JA, Mac Rae JC, Spence AM, Wilson S (1976) Intake and digestion of hill land vegetation by the red deer and the sheep. Nature 263, 763

Milne JA, Mac Rae JC, Spence AM, Wilson S (1978) A comparison of the voluntary intake and digestion of a range of forages at different times of the year by sheep and the red deer (Cervus elaphus). $\mathrm{Br}$ J Nutr 40, 347-356

Ortavant R, Bocquier F, Pelletier JP, Ravault JP, Thimonier J, Volland-Nail P (1988) Seasonality of reproduction in sheep and its control by photoperiod. Austr J Biol Sci 41, 69-85

Peters RR, Chapin LT, Leining KB, Tucker HA (1978) Supplemental lighting stimulates growth and lactation in cattle. Science 199, 911-912

Peters RR, Chapin LT, Emery RS, Tucker HA (1981) Milk yield, feed intake, prolactin, growth hormone and glucocorticoid response of cows to supplemented light. J Dairy Sci 64, 1671-1678

Russel AJF, Doney JM, Gunn RG (1969) Subjective assessment of body fat in live sheep. J Agric Sci Camb, 72, 451-454

Sitzia M, Roggero PP, Fois N, Molle G (1997) Grazing management of italian ryegrass sward with dairy sheep in the mediterranean environment. Proc XVII Int Grass Congr, 8- 19 June, Winnipeg, Canada (sous presse)

Sas (1988) SAS/STAT User's Guide, Release 6.03 Edition, Sas Institute Inc, Cary, NC, $1028 \mathrm{p}$

Tarttelin MF (1968) Cyclical variations in food and water intake in ewes. J Physiol 195, 29-31

Terqui M, Delouis C, Ortavant R (1983) Photoperiodism, hormones in sheep and goats. Curr Top Vet Med Anim Sci 26, 246-257

Yeates NTM (1949) The breeding season of the sheep with particular reference to its modification by artificial means using light. J Agric Sc Camb 39, 1-43 